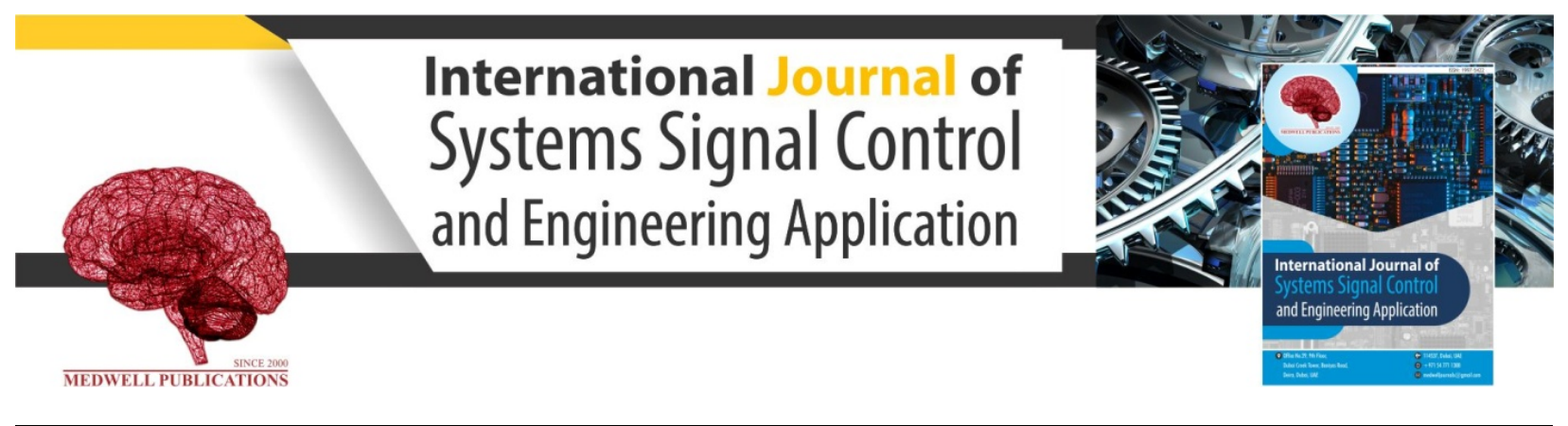

\title{
QPSK Signal Formulation Model using MATLAB Software
}

Rakhimov Temurbek Omonboyevich

Department of Telecommunication Technologies, Faculty Telecommunication Technologies and Professional Education, Urgench Branch of Tashkent, University of Information Technologies named after Muhammad Al-Khwarizmi, Khwarizm Region, Urgench City, Uzbekistan

Key words: Signal, system, modulation, amplitude, frequency, frequency bandwidth phase, spectrum, continuous signal, model

\section{Corresponding Author:}

Rakhimov Temurbek Omonboyevich

Department of Telecommunication Technologies, Faculty Telecommunication Technologies and Professional Education, Urgench Branch of Tashkent, University of Information Technologies named after Muhammad AlKhwarizmi, Urgench City, Khwarizm Region, Uzbekistan

Page No.: 21-25

Volume: 12, Issue 2, 2019

ISSN: 1997-5422

International Journal of Systems Signal Control and Engineering Application

Copy Right: Medwell Publications
Abstract: Radiovision systems are used to control different objects in the radio signal. On nonconverging systems, the signals are in the form of alterations in the amplitude, frequency and phase. In impulsive systems, the signal is in the form of a radio impulsion sequence (amplitude, phase, frequency band) and the number of impulses sequentially. In the study, QPSK signals were analyzed using MATLAB program which analyzed the QPSK module simultaneously with QPSK signals. In addition, QPSK modular modeling is also considered.

\section{INTRODUCTION}

The field of digital data transmission, phase modification and combinational modulation that is called squared is widespread. Modulation types in phase and amplitude-phase modulations are the most commonly used modulation types: BPSK, QPSK. Multiple signals are particularly spectacular in 4-phase: phase modulation (QPSK) and 16-square-meta Amplitude Modulation (16-QAM).

\section{MATERIALS AND METHODS}

Conception about QPSK signals: Signal transmitters are split into continuous impulse or digital radios. The QPSK modulated carrier has four different variations in the phase which are represented as symbols. Phases can accept $0, \pi / 2, \pi$ and $3 \pi / 2$ values. Each character represents two binary bitmaps.

Quadrature phase modulation, i.e., digital modulation or QPSK (Quadrature Phase Shift Key) binary phase digital modulated alarm phase change $(0,90,180$ and $270^{\circ}$ ). The sequence of transmitted bits is divided into sequence of pairs and null bits. The sequence of the same numbered bits produces duplicate pairs. This can be seen as complex bits, the actual batch of the bits is pure knots and the bundle forms the double bits. Complex bits are converted to impulses of +1 or -1 with $2 \mathrm{TC}$ duration.

\section{RESULTS AND DISCUSSION}

Mathematical form of QPSK signals: The mathematical expression of the QPSK signal can be written as follows: 
Int. J. Syst. Signal Control Eng. Appl., 12 (2): 21-25, 2019

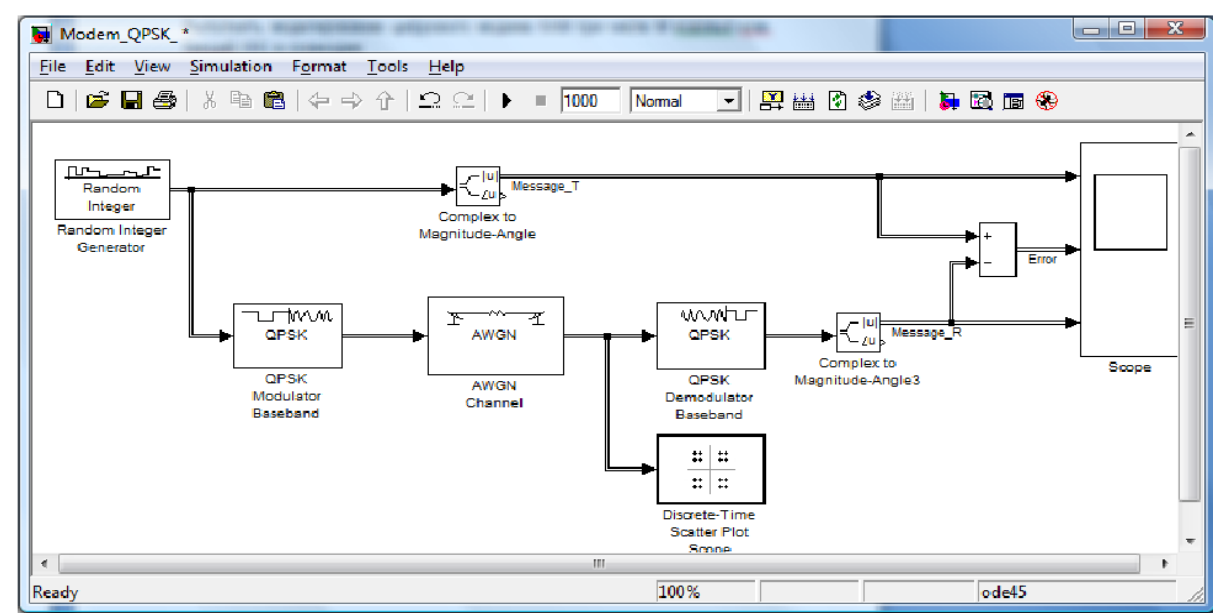

Fig. 1: Module of QPSK signaling (modem) device

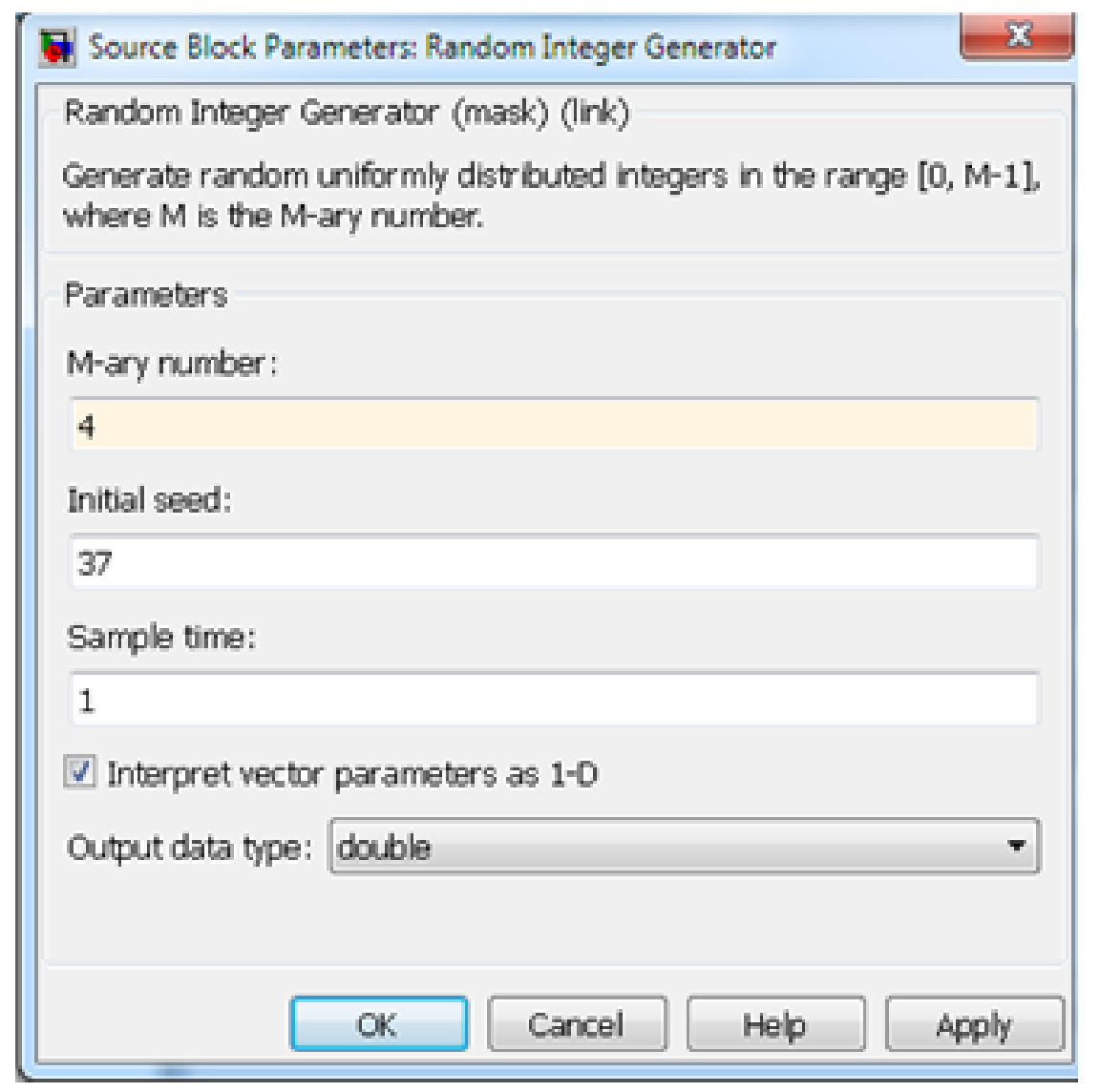

Fig. 2: Change input signal settings window

Where:

$$
\mathrm{S}(\mathrm{t})=\sqrt{\mathrm{I}^{2}+\mathrm{Q}^{2}} \cos \left(2 \pi \mathrm{f}_{0} \mathrm{t}-\varphi\right)
$$

$\varphi:$ The $\operatorname{arctg}(\mathrm{Q} / \mathrm{I})-$ phase of QPSK signals
I : The current values of the ship sequences of bits

Q : The pairs of binary ship values Expression of the QPSK signal: 
Int. J. Syst. Signal Control Eng. Appl., 12 (2): 21-25, 2019

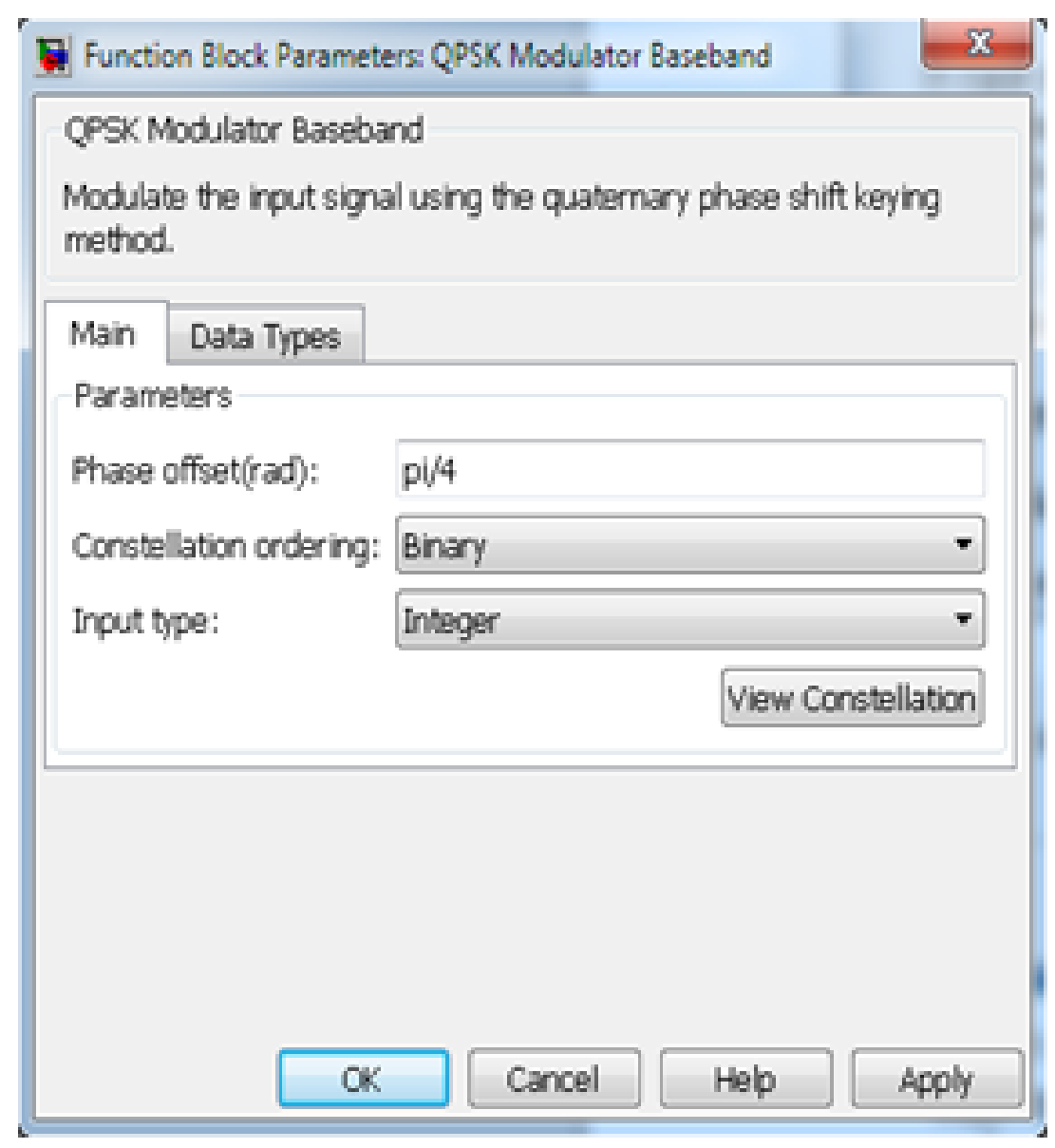

Fig. 3: Change the QPSK modulaer setting window

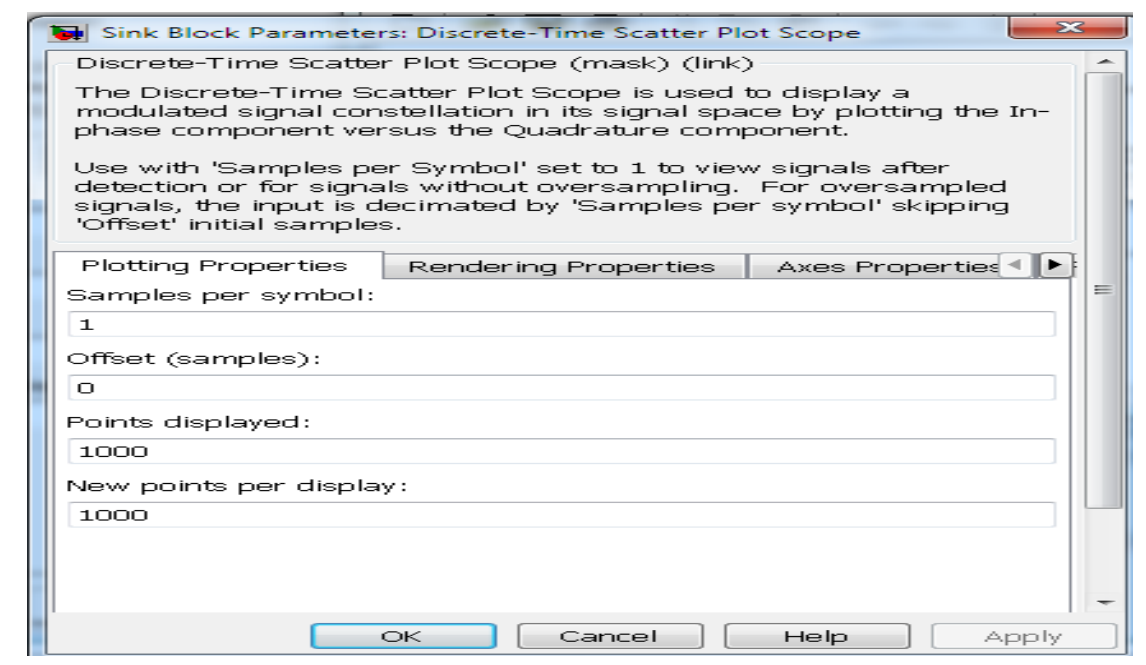

Fig. 4: Change the discrete device settings parameters window 

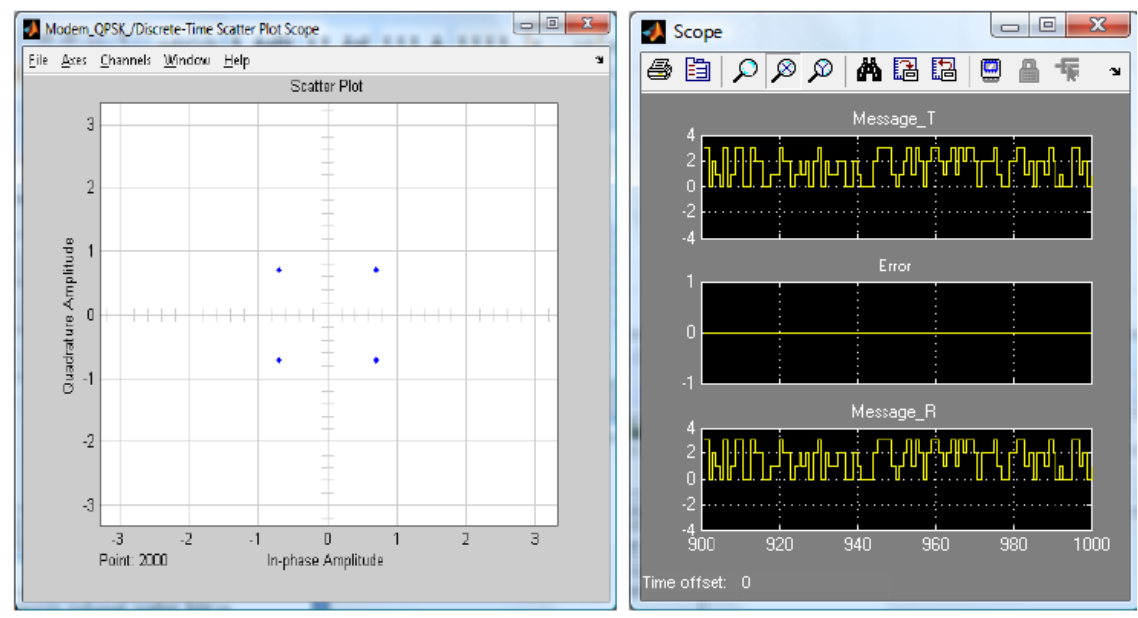

Fig. 5: The three different transmissions, received and noise signals
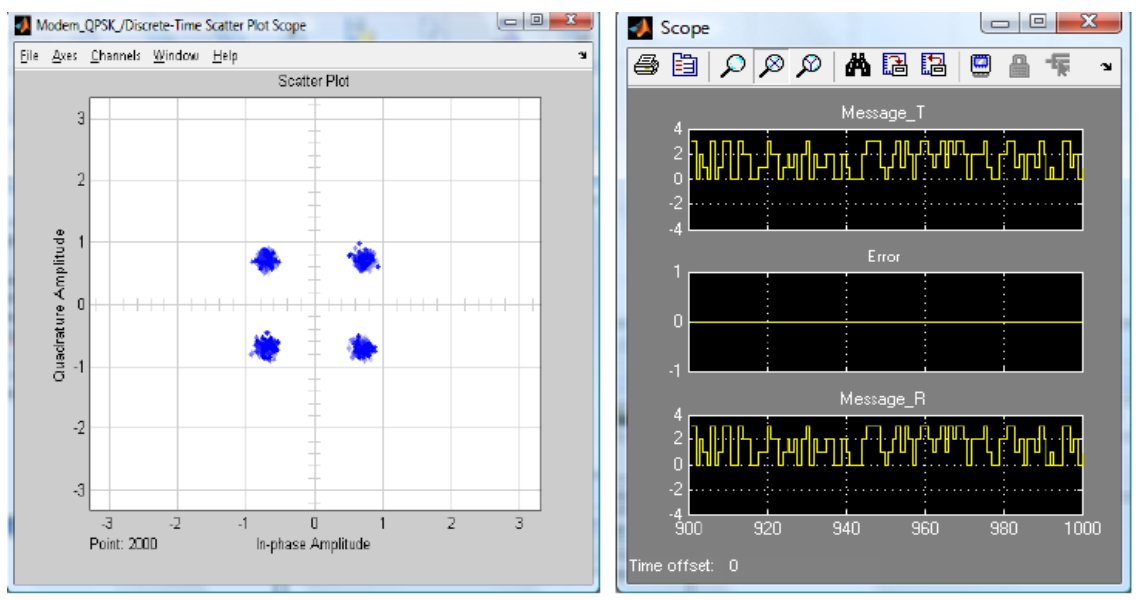

Fig. 6: Modified and noise feedback partially lost signal
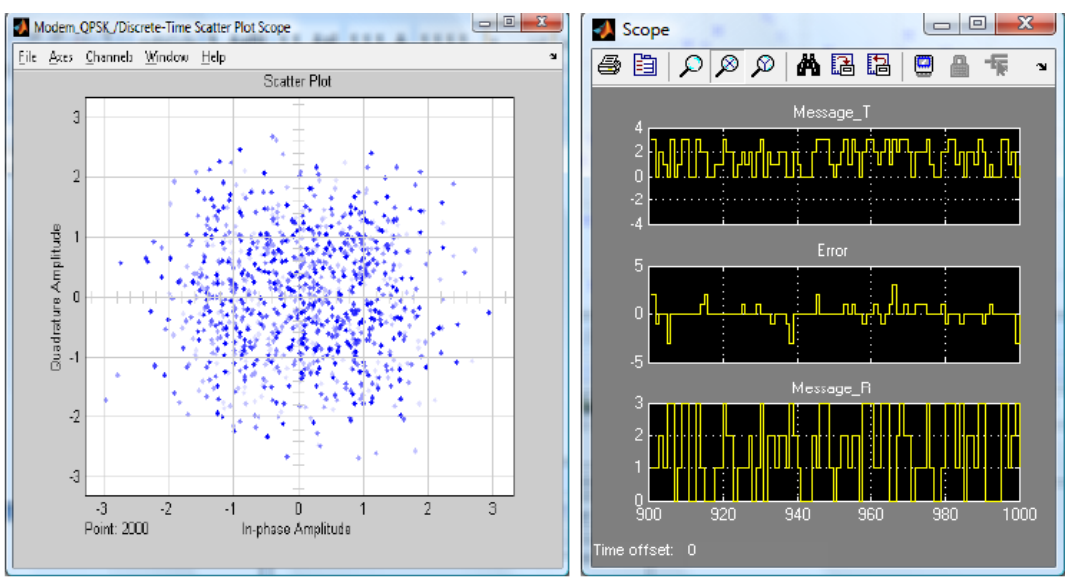

Fig. 7: Demodified signal and noise feedback 


$$
\mathrm{S}(\mathrm{t})=\sqrt{\mathrm{I}^{2}+\mathrm{Q}^{2}} \cos \left(2 \pi \mathrm{f}_{0} \mathrm{t}+\frac{\pi}{4}-\varphi\right)
$$

Appearance can be adjusted by 0, 90, 180 and $270^{\circ}$ (Proakis and Salehi, 2008; Miller, 1999). If we analyze the type of alarm we have seen above it will take a lot of time. If we consider this process in the Simulink section of MATLAB, it is enough to have just one inputinterface Fig. 1-7. The signal transmitted on digital systems is quantized (summed up) by time and stage. In each layer the signaling signal corresponding to the pulses is modulated. Digital systems can easily memorize, process and visualize images with exposure to exposure (Couch, 2012).

The novelty of the study also includes the process of analyzing the signal specification using MATLAB system of the Simulink section of the standard set of elements and blocks by Urkowitz (1983), Ziemer and Tranter (1976) and Whalen (1971).

By implementing these tasks, the user is expected to have QPSK signals closer to real-world conditions and the use of computer technology in practice will increase the interest of the student to his or her work and the experience will be faster and more accurate.

Moreover, due to the effective of information technology and distance learning, the modern education system is rising to a new stage of its development, i.e., the formation and development of the educational and informational environment.

\section{CONCLUSION}

It can be said that MATLAB program will help you to analyze the experimental skills of "Telecommunication technologies". It is impossible for the user to collect and use the radio-electronic devices in the home environment and this program can easily be used in home-based computing with the help of standard inputs and blocks of the Simulink section in MATLAB program and 60-70\% of them are able to consolidate their knowledge.

\section{REFERENCES}

Couch, L.W., 2012. Digital and Analog Communication Systems. 5th Edn., Macmillan Publisher, New York, USA.

Miller, S., 1999. Modulation with RF Carriers. MSc Thesis, Texas A\&M University, College Station, Texas.

Proakis, J.G. and M. Salehi, 2008. Digital Communications. 5th Edn., McGraw-Hill, New York, USA., ISBN: 9780071263788 , Pages: 1150.

Urkowitz, H., 1983. Signal Theory and Random Processes. Artech House, Norwood, Massachusetts, USA., ISBN:9780890061213, Pages: 715.

Whalen, A.D., 1971. Detection of Signals in Noise. Academic Press, Cambridge, Massachusetts, USA., Pages: 411.

Ziemer, R.E. and W.H. Tranter, 1976. Principles of Communications: Systems, Modulation and Noise. Houghton Mifflin Harcourt, Boston, Massachusetts, USA. 